\title{
Soft Metrics and EXIT Chart Analysis of Noncoherent MFSK with Diversity Reception
}

\author{
Sohail Ahmed ${ }^{1}$, Lie-Liang Yang ${ }^{2}$ and Lajos Hanzo ${ }^{2}$ \\ ${ }^{1}$ Dept. of Avionics, National University of Sciences \& Technology (NUST), Pakistan. \\ ${ }^{2}$ School of ECS, University of Southampton, SO17 1BJ, UK. \\ Tel: +44-23-80593125, Fax: +44-23-80594508 \\ Email: ${ }^{1}$ sohailahmed71@ @otmail.com, ${ }^{2}\{11 y, l h\} @$ ecs.soton.ac.uk; http://www-mobile.ecs.soton.ac.uk
}

\begin{abstract}
A convolutionally encoded noncoherent $M$-ary Frequency Shift Keying (MFSK) modulation scheme using diversity reception is considered. A novel technique of generating soft information for Soft Decision Decoding (SDD) of noncoherent MFSK is derived assuming various channel models. Furthermore, the convergence behavior of the MFSK receiver is investigated using EXtrinsic Information Transfer (EXIT) charts. It is demonstrated that the expressions derived for SDD of the MFSK scheme are useful for exploiting the beneficial effects of diversity. We also demonstrate that EXIT chart analysis provides accurate insights into the system's iterative decoding behavior.
\end{abstract}

\section{INTRODUCTION}

Noncoherent $M$-ary Frequency Shift Keying (MFSK) constitutes an attractive, low-complexity modulation technique, especially cooperative communications systems, since it does not require channel estimation. This beneficial characteristic lends simplicity to the receiver and also results in an attractive performance in fast fading channels [1]. Moreover, by employing diversity combining at the receiver, the multipath fading encountered in wireless channels may be combatted by achieving a substantial diversity gain. Hence, noncoherent MFSK combined with diversity reception has attracted a lot of attention in the context of wireless communication [2], [3]. A range of further MFSK-related research problems were considered in [4], [5]. Fast frequency hopping may be deemed as a special case of diversity-aided MFSK [6], [7].

Soft Decision Decoding (SDD) of noncoherent MFSK has also been investigated and useful metrics have been proposed for computing soft information from the received signal [8][13]. The concept of extrinsic information exchange between an $M$-ary orthogonal demodulator and a Soft-In-Soft-Out (SISO) decoder has been investigated in [6], [14]. In [6], further insights have been provided with the aid of EXtrinsic Information Transfer (EXIT) chart analysis. EXIT charts constitute useful semi-analytical tools, which were proposed by ten Brink [15] for analyzing the convergence behavior of iteratively decoded schemes. In the open literature, little attention has been devoted to the investigation of SISO decoding in noncoherent MFSK-based schemes exploiting diversity reception. The reasons for this trend might be the difficulty of deriving soft information from the $M$-dimensional signal received through diverse paths as well as the challenge of the

The financial support of Higher Education Commission, Pakistan as well as of the EU's Optimix project and of the EPSRC UK is gratefully acknowledged. subsequent exploitation of the soft information forwarded by the SISO decoder to the MFSK demodulator.

In this contribution, we derive simplified but optimum soft metrics from the received MFSK signal exploiting $L$ th order diversity, assuming various channel models. More specifically, we derive soft information for Additive White Gaussian Noise (AWGN), Rayleigh and Nakagami- $m$ fading channels. Moreover, we investigate the convergence behavior of the noncoherent MFSK receiver with the aid of EXIT charts.

The rest of this paper is structured as follows. In Sec. II, the system considered is described, while in Sec. III the soft metrics are derived and the Iterative Decoding (ID) process is discussed. In Sec. IV, our EXIT chart analysis of the MFSK scheme is presented and our simulation-based Bit Error Ratio (BER) results are discussed. Finally, in Sec. V we present our conclusions.

\section{System OVERVIEW}

A convolutionally encoded noncoherent MFSK modulation scheme is considered, which may be deemed as a type of Bit Interleaved Coded Modulation (BICM) scheme [6]. The data to be transmitted are first encoded by a binary nonrecursive, Non-Systematic Convolutional (NSC) [16] code before bit interleaving. Following bit interleaving, the NSC coded bits are converted to $M$-ary symbols, using the relation: $n=\sum_{i=0}^{\log _{2}(M)-1} b_{i} 2^{i}$, where $n=0,1, \ldots, M-1$ is the $M$ ary symbol and $b_{i}$ denotes the bit in the $i$ th position. In the MFSK modulator, an MFSK tone is chosen for transmission corresponding to each $M$-ary symbol [1].

The channel is assumed to be a frequency-selective fading medium for each of the transmitted frequencies. Hence, the transmitted signal is received via $L$ diverse paths, which we assume to be independent and uncorrelated. We consider three different models for the channel, namely the AWGN, the Rayleigh fading model and the Nakagami- $m$ model.

The receiver schematic is shown in Fig. 1 in conjunction with Fig. 2, where the demodulator is comprised of $M$ branches, each corresponding to a single MFSK tone and consisting of a bandpass filter (BPF), a square-law detector [1] as well as a diversity combiner, which performs linear or equal gain combining [3] of the signals received via diverse paths. Since MFSK is a noncoherent scheme, we consider the case of equal gain combining, which results in a simpler receiver structure than that required by optimum maximal ratio combining [17]. 


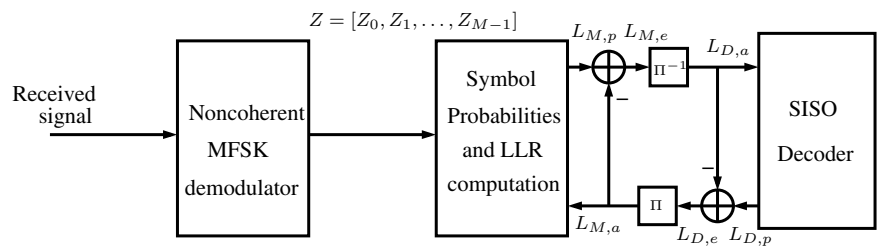

Fig. 1. Block diagram of the receiver consisting of the serial concatenation of noncoherent MFSK demodulator and SISO channel decoder, where MFSK demodulator is as shown in Fig. 2

The $l$ th path's signal at the input of this receiver can be expressed as

$$
r_{l}(t)=\alpha_{l} \sqrt{2 P} \cos \left\{2 \pi f_{n} t+\phi_{n}\right\}+n(t),
$$

where $P$ is the power of the transmitted signal, $f_{n}$ is the $n$th MFSK tone's frequency and $\phi_{n}$ is the phase resulting from the joint effects of MFSK modulation and the channel's phase rotation. Moreover, $n(t)$ is the AWGN having a singlesided power spectral density of $N_{0}$, while $\alpha_{l}$ is the channel's amplitude attenuation factor, which may be either Rayleigh or Nakagami- $m$ distributed, since both types of channel models are considered in this paper. For a non-fading channel, we have $\alpha_{l}=1$.

The decision variable recorded after diversity combining, as seen in Fig. 2, is given by

$$
Z_{n}=\sum_{l=0}^{L-1} U_{n l}, \quad n=0,1, \ldots, M-1 .
$$

where $U_{n l}$ represents the square-law detector's output [1], seen in Fig. 2, corresponding to the $n$th tone in the $l$ th path. At the output of the diversity combiner, the corresponding symbol probabilities and Log-Likelihood Ratios (LLRs) are computed, as explained in the following section, which are then fed to the channel decoder of Fig. 1.

Notations: In our discussions we use the notations $P_{M, a}$, $P_{M, p}$ and $P_{M, e}$ for the a priori, a posteriori and the extrinsic symbol probabilities of the demodulator, respectively. The corresponding notations for the channel decoder are $P_{D, a}$, $P_{D, p}$ and $P_{D, e}$. Likewise, $L_{M, a}, L_{M, p}$ and $L_{M, e}$ denote the a priori, a posteriori and extrinsic LLRs of the demodulator, while $I_{M, a}, I_{M, p}$ and $I_{M, e}$ represent the associated Mutual Information (MI). The corresponding metrics for the outer decoder are denoted by $L_{D, a}, L_{D, p}$ and $L_{D, e}$ and $I_{D, a}, I_{D, p}$ and $I_{D, e}$.

\section{ITERATIVE DECODING PROCESS}

In this section we discuss how soft information is derived from the channel's output observations and how ID is carried out by exchanging MI between the demodulator and the outer decoder seen in Fig. 1. We consider three different channel models and derive expressions for the symbol probabilities in each case.

In order to compute the LLRs, we need the probability that the $n$th symbol or FSK tone was transmitted, $n=$ $0, \ldots, M-1$, given that the signal $\mathbf{Z}=\left[Z_{0}, Z_{1}, \ldots, Z_{M-1}\right]$,

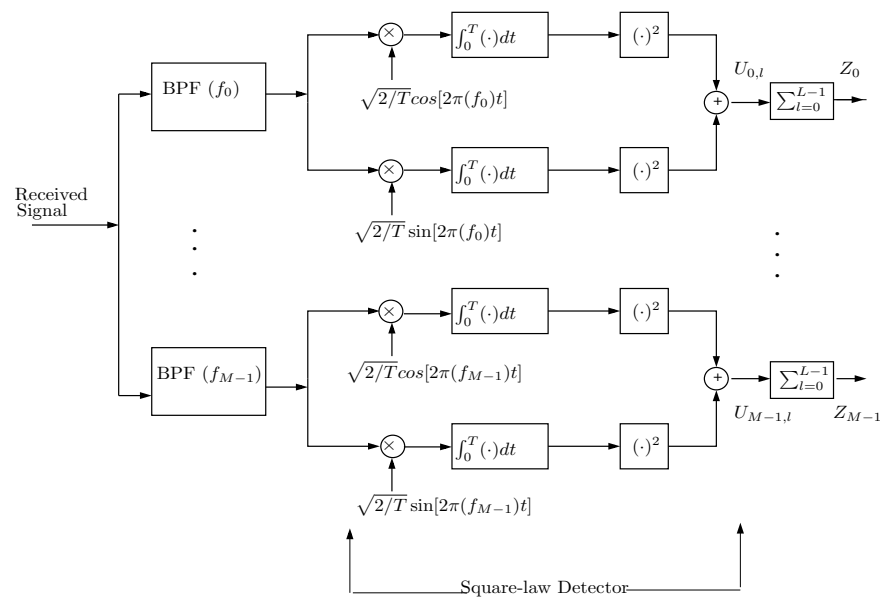

Fig. 2. Block diagram of a noncoherent MFSK Receiver using equal gain combining

which consists of the $M$ diversity combiner outputs seen in Figs. 1 and 2, is received. This probability is given by [6], [7]

$$
P_{M, p}(n \mid \mathbf{Z})=\frac{p(\mathbf{Z} \mid n) P(n)}{p(\mathbf{Z})},
$$

where $p(\mathbf{Z} \mid n)$ is the Probability Density Function (PDF) of the received signal $\mathbf{Z}$, given that the $n$th tone is transmitted. Furthermore, $P(n)$ is the a priori probability of the symbol $n$, while $p(\mathbf{Z})=\sum_{n=0}^{M-1} p(\mathbf{Z} \mid n) P(n)$ is the PDF of the received signal set $\mathbf{Z}$, which is the same, regardless of the value of $n$. Moreover, for equiprobable symbols, we have $P(n)=1 / M$. Hence, the PDF $p(\mathbf{Z} \mid n)$ constitutes sufficient statistics required for marginalizing the probability $P_{M, p}(n \mid \mathbf{Z})$. For independent fading of all tones, the PDF $p(\mathbf{Z} \mid n)$ is given by [6], [7]

$$
p(\mathbf{Z} \mid n)=f_{Z_{n}}\left(x_{n} \mid n\right) \prod_{j=0, j \neq n}^{M-1} f_{Z_{n}}\left(x_{j} \mid n\right),
$$

where $f_{Z_{j}}\left(x_{j} \mid n\right)$ represents the PDF of the $j$ th diversity combiner output, $j=0,1, \ldots, M-1$, given that the $n$th tone is transmitted.

Soft Information in Non-fading Channel In the absence of fading and when only AWGN is present, the linear combiner's output is non-central Chi-square distributed with $2 L$ degrees of freedom [1]. Consequently, the PDF of the noisenormalized combiner output, assuming that the $n$th tone, $n=$ $0,1, \ldots, M-1$, is transmitted, may be expressed as

$$
f_{Z_{n}}\left(x_{n} \mid n\right)=\left(\frac{x_{n}}{\gamma}\right)^{(L-1) / 2} e^{-\left(x_{n}+\gamma\right)} I_{L-1}\left(2 \sqrt{x_{n} \gamma}\right),
$$

while for the non-signal tones, we have

$$
f_{Z_{j}}\left(x_{j} \mid n\right)=\frac{x_{j}^{L-1}}{\Gamma(L)} e^{-x_{j}}
$$

where $\gamma=\gamma_{h} \sum_{l=0}^{L-1} \alpha_{l}^{2}, \gamma_{h}=R_{c} E_{b} \log _{2} M /\left(L N_{0}\right)$ is the Signal-to-Noise Ratio (SNR) per diversity path, $R_{c}$ is the code rate and $E_{b}$ is the transmitted energy per bit. Moreover, in (5), $I_{L-1}($.$) is the modified Bessel function of the first kind of$ 
$(L-1)$ th order, while in $(6), \Gamma()$ is the Gamma function [18]. Upon inserting (5) and (6) into (4), we have

$$
\begin{aligned}
p(\mathbf{Z} \mid n) & =\left(\frac{x_{n}}{\gamma}\right)^{(L-1) / 2} e^{-\left(x_{n}+\gamma\right)} I_{L-1}\left(2 \sqrt{x_{n} \gamma}\right) \\
& \times \prod_{j=0, j \neq n}^{M-1} \frac{x_{j}^{L-1}}{\Gamma(L)} e^{-x_{j}} \\
& \left.=\left[\frac{e^{-\gamma}}{[(L-1) !]^{M-1} \gamma^{(L-1) / 2}}\right] \prod_{j=0}^{M-1}\left[x_{j}^{L-1} e^{-x_{j}}\right]\right] \\
& \times I_{L-1}\left(2 \sqrt{x_{n} \gamma}\right) x_{n}^{-(L-1) / 2} .
\end{aligned}
$$

We see in (7) that for any of the $n$th symbol, $n=$ $0,1, \ldots, M-1$, all the terms except for the last two terms are common. Consequently, ignoring the common terms as a normalization factor, the normalized PDF $p(\mathbf{Z} \mid n)$ for the AWGN channel may be expressed as

$$
p(\mathbf{Z} \mid n)=I_{L-1}\left(2 \sqrt{x_{n} \gamma}\right) x_{n}^{-(L-1) / 2} .
$$

Soft information in Nakagami- $m$ Fading Channel In this case, the linear combiner's output is non-central Chi-square distributed with $2 L$ degrees of freedom [1], but conditioned on the received SNR $\gamma$. Consequently, the PDF of the noisenormalized combiner output may be expressed as

$$
f_{Z_{n}}\left(x_{n} \mid \gamma, n\right)=\left(\frac{x_{n}}{\gamma}\right)^{(L-1) / 2} e^{-\left(x_{n}+\gamma\right)} I_{L-1}\left(2 \sqrt{x_{n} \gamma}\right) .
$$

For the $j$ th non-signal tone, $j=0,1, \ldots, M-1, j \neq n$, the linear combiner's output will be repeated for convenience in (5). In order to derive the unconditional PDF of $Z_{n}$, the expression given by (9) must be averaged over the PDF of $\gamma$, which can be derived from the PDF of $\alpha_{l}$ discussed above in the context of (1). Since $\alpha_{l}$ is Nakagami- $m$ distributed, its $\mathrm{PDF}$ is given by [1]

$$
f_{\alpha_{l}}(t)=\frac{2 t^{2 m-1}}{\Gamma(m)}\left(\frac{m}{\Omega}\right)^{m} e^{-m t^{2} / \Omega},
$$

where $m$ is the Nakagami fading parameter, and $\Omega=E\left[\alpha_{l}^{2}\right]$. Consequently, it can be shown that $\gamma_{l}=\gamma_{h} \alpha_{l}^{2}$ is Gamma distributed and its PDF can be readily derived, with the aid of [1], yielding

$$
f_{\gamma_{l}}(r)=\frac{r^{m-1}}{\Gamma(m)}\left(\frac{m}{\gamma_{h}}\right)^{m} e^{-m r / \gamma_{h}}
$$

The Characteristic Function (CF) of the PDF in (11) may be readily expressed as [1]

$$
\phi_{\gamma_{l}}(\omega)=\frac{1}{\left(1-\mathcal{J} \omega \gamma_{h} / m\right)^{m}},
$$

where $\mathcal{J}=\sqrt{-1}$. Since the signals in all hops are i.i.d. the CF of $\gamma=\sum_{l=0}^{L-1} \gamma_{l}$ is given by [1]

$$
\phi_{\gamma}(\omega)=\frac{1}{\left(1-\mathcal{J} \omega \gamma_{h} / m\right)^{m L}} .
$$

From the above $\mathrm{CF}$, we can derive the PDF of the random variable $\gamma$, which is given by [1]

$$
f_{\gamma}(\gamma)=\frac{\gamma^{m L-1}}{\Gamma(m L)}\left(\frac{m}{\gamma_{h}}\right)^{m L} e^{-m \gamma / \gamma_{h}}
$$

Consequently, from (9) and (14), we have

$$
\begin{aligned}
f_{Z_{n}}\left(x_{n} \mid n\right) & =\int_{0}^{\infty} f_{Z_{n}}\left(x_{n} \mid \gamma, n\right) f_{\gamma}(\gamma) d \gamma \\
& =\int_{0}^{\infty}\left(\frac{x_{n}}{\gamma}\right)^{\frac{(L-1)}{2}} e^{-\left(x_{n}+\gamma\right)} I_{L-1}\left(2 \sqrt{x_{n} \gamma}\right) \\
& \times \frac{\gamma^{m L-1}}{\Gamma(m L)}\left(\frac{m}{\gamma_{h}}\right)^{m L} e^{-m \gamma / \gamma_{h}} d \gamma
\end{aligned}
$$

Upon applying a suitable change of variable, the above equation may be expressed as [19]

$$
\begin{aligned}
f_{Z_{n}}\left(x_{n} \mid n\right) & =x_{n}^{L-1} e^{-x_{n}} \frac{1}{\Gamma(L)}\left(\frac{m}{m+\gamma_{h}}\right)^{m L} \\
& \times \Phi\left(m L, L, \frac{\gamma_{h} x_{n}}{m+\gamma_{h}}\right)
\end{aligned}
$$

where $\Phi(x, y, z)$ is the hypergeometric function [19]. Note that for $m=1$, it can be shown with the aid of the relation $\Phi\left(L, L, \frac{\gamma_{h} x_{n}}{1+\gamma_{h}}\right)=\exp \left(\frac{\gamma_{h} x_{n}}{1+\gamma_{h}}\right)$ [19] that (16) reduces to the corresponding expression derived for Rayleigh fading channels in [7] and given by (20) below.

Inserting (16) and (5) into (4), and after a few minor simplifications, we arrive at:

$$
\begin{aligned}
p(\mathbf{Z} \mid n) & =\left[\prod_{j=0}^{M-1}\left[\frac{x_{j}^{L-1} e^{-x_{j}}}{\Gamma(L)}\right]\left(\frac{m}{m+\gamma_{h}}\right)^{m L}\right] \\
& \times \Phi\left(m L, L, \frac{\gamma_{h} x_{n}}{m+\gamma_{h}}\right) .
\end{aligned}
$$

Again, in (17), for any of the $n$th symbol, $n=0,1, \ldots, M-$ 1 , all the terms except for the hypergeometric function term are common. Consequently, when considering the common terms as normalization factors and hence ignoring them, the normalized PDF $p(\mathbf{Z} \mid n)$ for the Nakagami- $m$ channel may be expressed as ${ }^{1}$

$$
p(\mathbf{Z} \mid n)=\Phi\left(m L, L, \frac{\gamma_{h} x_{n}}{m+\gamma_{h}}\right) .
$$

Soft Information in Rayleigh Fading Channel In [7], using the PDFs and the CFs [1] of the square-law detector outputs, we derived a simplified expression for the $p(\mathbf{Z} \mid n)$ of (4) for the Rayleigh fading channel given by

$$
p(\mathbf{Z} \mid n)=\exp \left(\frac{x_{n} \gamma_{h}}{1+\gamma_{h}}\right) \text {. }
$$

Upon inserting (8), (19) or (20) in (3) and ignoring the common terms, we can derive the corresponding normalized symbol probabilities for each of the channel models

${ }^{1}$ For ease of computation, the hypergeometric function may be written in the form of an infinite series given by [19]

$$
\begin{aligned}
\Phi\left(m L, L, \frac{\gamma_{h} x_{n}}{m+\gamma_{h}}\right) & =\Phi\left(L(1-m), L, \frac{-\gamma_{h} x_{n}}{m+\gamma_{h}}\right) \\
& =\sum_{i=0}^{\infty} \frac{(L(1-m))_{i}}{(L)_{i} i !}\left[\frac{-\gamma_{h} x_{n}}{m+\gamma_{h}}\right]^{i}
\end{aligned}
$$

where $(a)_{i}=a(a+1) \ldots(a+i-1)=(a+i-1) ! /(a-1) !$ is the Pochammer function [19]. For negative integers, we have $(-a)_{i}=(-1)^{i}(a) ! /(a-i) !$. Note that for $m>1$, the infinite series is reduced to a finite one, consisting of $L(m-1)$ terms. 
considered. Finally, considering the bits-to-symbols mapping assumed in Sec. II, the bit probabilities can be derived from the symbol probabilities using the relation [14], [20]:

$$
P_{M, p}\left(b_{i}=0 \mid \mathbf{Z}\right)=\sum_{t=0}^{M / 2-1} P_{M, p}(n \mid \mathbf{Z}), \quad n=t+\left\lfloor\frac{t}{2^{i}}\right\rfloor 2^{i},
$$

where $\lfloor x\rfloor$ represents the largest integer less than or equal to $x$. Consequently, the LLRs are computed from the bit probabilities using the relation [15], [20]

$$
L_{M, p}(i \mid \mathbf{Z})=\log \left[\frac{P_{M, p}\left(b_{i}=1 \mid \mathbf{Z}\right.}{P_{M, p}\left(b_{i}=0 \mid \mathbf{Z}\right.}\right] .
$$

After subtracting the a priori LLRs, the extrinsic LLRs may be fed to the decoder as seen in Fig. 1. The decoder outputs a posteriori information, which may be fed back to the demodulator as its a priori information. In conventional ID, this exchange of soft information between the demodulator and decoder may be invoked a number of times [16].

In the foregoing analysis, we noted that the knowledge of the received SNR is required to compute symbol probabilities from (8), (19) and (20). In noncoherent systems, accurate estimation of the channel may not be practicable. However, the SNR may be estimated to a reasonable degree of accuracy by computing a running average of the received AWGN power over a number of say 20 received samples. We assume in this contribution that the received SNR is accurately known at the receiver. We also note that by inserting $L=1$ in (8) and (19), we arrive at the corresponding expressions for the MFSK scheme dispensing with EG diversity combining.

\section{EXIT ChaRT ANALYSis AND Simulation RESUlts}

Having derived the expressions characterizing the soft information passed by the demodulator to the decoder, let us now study the MI transfer characteristics of the MFSK demodulator using EXIT curves [15], when it is serially concatenated with an outer SISO decoder, as shown in Fig 1. We assume that the a priori information may be sufficiently accurately modeled by a Gaussian distribution, as discussed in [15]. Moreover, all MI measurements were made using the histogram-based approximation of the true distribution [15]. Furthermore, unless otherwise stated, we employ a $1 / 2$ rate NSC code with constraint length of 3 characterized by the octally represented generator polynomials of $(7,5)$ and the coded bits are interleaved by a 24000-bit random interleaver $\pi$.

The MI transfer characteristics of the MFSK demodulator recorded for transmission over a Nakagami- $m$ fading channel having a fading parameter of $m=3$ is shown in Fig. 3 for various $M$ values. This figure demonstrates that for $M=2$, i.e. for binary FSK (BFSK), the EXIT curve is a horizontal line, indicating that the BFSK demodulator will yield no improvement in its output extrinsic MI upon increasing the a priori MI. Thus, no Iterative Gain (IG) is expected from the BFSK demodulator, if it is employed as depicted in Fig. 1. By contrast, as shown in Fig. 3, for higher $M$ values the EXIT curves emerge from a higher point on the $Y$-axis and they are also steeper. This observation indicates that a higher IG is

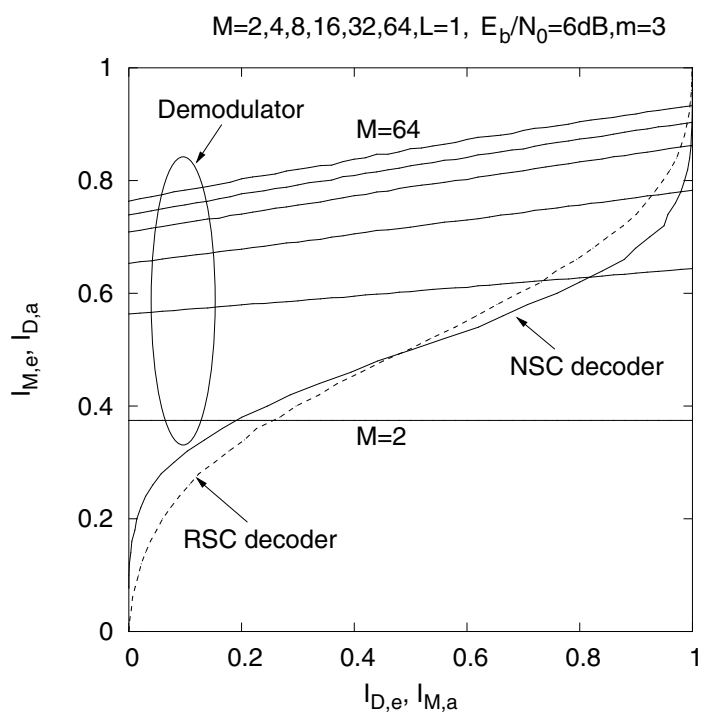

Fig. 3. EXIT characteristics of a MFSK demodulator and SISO decoder in Nakagami- $m$ fading channel, assuming various $M$ values.

expected from the MFSK demodulator, when using a higher $M$ value. Furthermore, in Fig. 3, the EXIT curves of both the NSC and of the Recursive Systematic Convolutional (RSC) codes are shown, with the axes inverted. We observe that the EXIT curve of the RSC decoder is steeper than that of the NSC decoder in the range of 0.2 to 0.8 . Hence, the demodulator's EXIT curve intersects the RSC decoder's curve at a lower MI value, indicating that due to the low-gradient nature of the MFSK demodulator's EXIT curves, this modulation scheme may be more beneficially concatenated with the NSC code than with the RSC code.

The effects of the SNR on the evolution of the demodulator's MI are shown in Fig. 4. As expected, at increased $E_{b} / N_{0}$ values the MI curve emerges from a higher value on the $Y$ axis at zero abscissa. However, the slope of the MI curve is reduced as the SNR is increased, implying a lack of IG.

In Fig. 5, the BER versus SNR performance of the SDD scheme invoking various numbers of ID iterations $N_{I D}$ between the demodulator and the outer decoder, as well as that of the Hard Decision Decoding (HDD) based scheme has been shown. The results of Fig. 5 demonstrate that the SDD scheme using $N_{I D}=0$ iterations outperforms the HDD by about $2 \mathrm{~dB}$ at $\mathrm{BER}=10^{-3}$, validating the soft information derived in Sec. III. The results of this figure also demonstrate that an overall gain of about $1.7 \mathrm{~dB}$ is obtained at $\mathrm{BER}=10^{-5}$ by invoking $N_{I D}=2$ iterations, although only a modest IG is achieved during the 2 nd iteration. Moreover, we observe that the NSC code remains superior to its RSC counterpart, thus confirming the accuracy of our EXIT chart analysis seen in Fig. 3.

\section{CONCLUSION}

In this contribution, we have derived expressions for computing the soft information from an MFSK signal for the sake of serial concatenation and mutual information exchange between a noncoherent MFSK receiver exploiting diversity combining and an outer SISO decoder. The soft information was derived assuming three channels models, namely the 


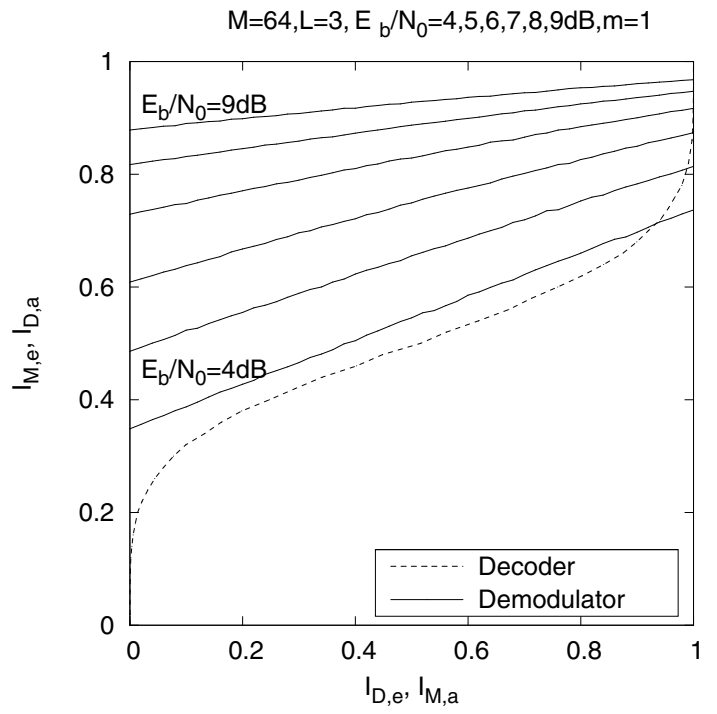

Fig. 4. EXIT characteristics of a MFSK demodulator and SISO NSC decoder in Nakagami- $m$ fading channel, assuming various $E_{b} / N_{0}$ values.

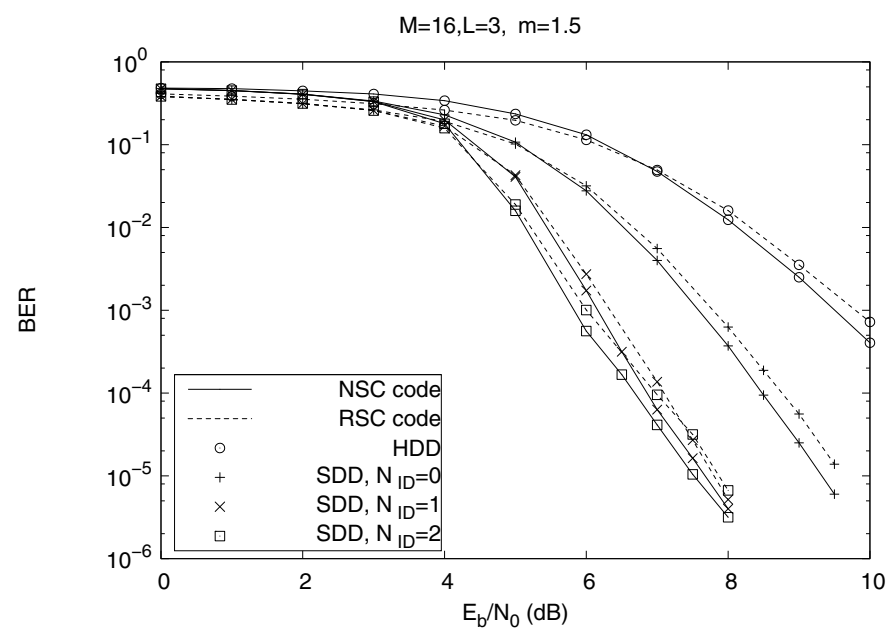

Fig. 5. BER performance comparison of HDD and SDD of an MFSK demodulator in Nakagami- $m$ fading channel.

noise-only, the Nakagami- $m$ model and the Rayleigh fading model, thus enabling the SDD of the MFSK based schemes to benefit from diversity combining. The expressions derived are straightforward to compute, although they need the knowledge of the received SNR. Although we characterized our scheme using a convolutional code, the expressions derived for the soft information may be employed for MFSK concatenated with arbitrary channel coding schemes.

We also carried out the EXIT chart analysis of the MFSK receiver and investigated the influence of various parameters on the achievable IG. It was revealed that increasing the modulation order results in an improved performance as well as in a healthier IG in case of the non-coherent MFSK system considered. Hence, when designing an MFSK based scheme, higher $M$ values may be preferred. Another observation made using our EXIT chart analysis is that the MFSK scheme may be more beneficially concatenated with an NSC code rather than an RSC code.

\section{REFERENCES}

[1] J. G. Proakis, Digital communications. Singapore: Mcgraw-Hill, 2001.

[2] Q. T. Zhang, "Exact analysis of postdetection combining for DPSK and NFSK systems over arbitrarily correlated Nakagami channels," IEEE Transactions on Communications, vol. 46, pp. 1459-1467, June 1998.

[3] N. C. Beaulieu and A. A. Abu-Dayya, "Analysis of equal gain diversity on Nakagami fading Channels," IEEE Transactions on Communications, vol. 39, pp. 225-234, February 1991.

[4] S. Ahmed, L-L. Yang, L. Hanzo: Mellin-Transform-Based Performance Analysis of FFH $M$-ary FSK Using Product Combining for Combatting Partial-Band Noise Jamming, IEEE Transactions on Vehicular Technology, Volume 57, Issue 5, Sept. 2008, pp 2757 - 2765

[5] S. Ahmed, L-L. Yang, L. Hanzo: Erasure Insertion in RS-Coded SFH MFSK Subjected to Tone Jamming and Rayleigh Fading, IEEE Transactions on Vehicular Technology, Volume 56, Issue 6, Part 1, Nov. 2007 pp 3563 - 3571

[6] M. C. Valenti, E. Hueffmeier, B. Bogusch, and J. Fryer, "Towards the capacity of noncoherent orthogonal modulation: BICM-ID for turbo coded NFSK," in IEEE Conference on Military Communications, 2004. MILCOM 2004, vol. 3, pp. 1549 - 1555, October-November 2004.

[7] S. Ahmed, S. X. Ng, L. L. Yang, and L. Hanzo, "Iterative Decoding and Soft Interference Cancellation in Fast Frequency Hopping Multiuser System Using Clipped Combining," in Proceedings of the IEEE Wireless Communications and Networking Conference, WCNC 2007., pp. 723 728, March 2007.

[8] W. E. Stark, "Coding for frequency-hopped spread-spectrum communication with partial-band interference-part I: capacity and cutoff rate," IEEE Transactions on Communications, vol. COM-33, pp. 1036-1044, October 1985.

[9] K. Cheun and W. E. Stark, "Performance of robust metrics with convolutional coding and diversity in FHSS systems under partial-band noise jamming," IEEE Transactions on Communications, vol. 41, pp. 200 209, January 1993.

[10] B. M. Peric, M. R. Souryal, E. Larsson, and B. R. Vojcic, "Soft Decision Metrics for Turbo-coded FH M-FSK Ad Hoc Packet Radio Networks," in IEEE 61st Vehicular Technology Conference, Spring 2005, vol. 2, pp. 724 - 727, May-June 2005.

[11] C. Durand, J. Boutros, and E. Bejjani, "Forward error correction of FSK alphabets for noncoherent transmissions over AWGN channel," IEEE Communications Letters, vol. 4, pp. 318-320, October 2000.

[12] N. Chayat, "Turbo codes for incoherent M-ary orthogonal signaling," in Nineteenth Convention of Electrical and Electronics Engineers in Israel, 1996., vol. 3, pp. 471-474, November 1996.

[13] J. S. G. Panaro, "Simplified Soft-Output Demapper for Bit-Interleaved Coded Orthogonal Modulation," in International Conference on Wireless and Mobile Communications, 2006. ICWMC '06., pp. 71-71, June 2006.

[14] P. C. P. Liang and W. E. Stark, "Algorithm for joint decoding of turbo codes and M-ary orthogonal modulation," in IEEE International Symposium on Information Theory, 2000, p. 191, June 2000.

[15] S. ten Brink, "Convergence of iterative decoding," IEEE Transactions on Communications, vol. 49, pp. 1727 - 1737, October 2001.

[16] L. Hanzo, T. H. Liew, and B. L. Yeap, Turbo Coding, Turbo Equalisation and Space-Time Coding for Transmission over Fading Channels. England: John Wiley and Sons, England, 2002.

[17] G. L. Turin, "On optimal diversity reception, II," IEEE Transactions on Communications, vol. 10, pp. 22-31, March 1962

[18] M. Abramowitz and I. A. Stegun, Handbook of mathematical functions. New York: Dover Publications, Inc., 1970.

[19] I. Gradshteyn and I. M. Ryzhik, Tables of integrals, series and products. London: Academic Press, 1965.

[20] U. C. Fiebig, "Soft-decision and erasure decoding in fast frequencyhopping systems with convolutional, turbo, and Reed-Solomon codes," IEEE Transactions on Communications, vol. 47, pp. 1646 - 1654, November 1999. 